\title{
Diagnostic value of echocardiography on detecting the various types of anomalous origin of the left coronary artery from the pulmonary artery
}

\author{
Yi Yu' ${ }^{1}$, Qun-Shan Wang ${ }^{1}$, Xi-Fang Wang ${ }^{2}$, Jian Sun ${ }^{1}$, Ling-Wei Yu ${ }^{3}$, Ming Ding ${ }^{3}$, Yi-Gang Li ${ }^{1}$ \\ ${ }^{1}$ Department of Cardiology, ${ }^{2}$ Department of Performance, ${ }^{3}$ Department of Radiology, Xinhua Hospital Affiliated with the School of Medicine, \\ Shanghai Jiao Tong University, Shanghai 200092, China \\ Contributions: (I) Conception and design: Y Yu; (II) Administrative support: YG Li; (III) Provision of study materials or patients: QS Wang, LW Yu; (IV) \\ Collection and assembly of data: J Sun, M Ding; (V) Data analysis and interpretation: XF Wang; (VI) Manuscript writing: All authors; (VII) Final \\ approval of manuscript: All authors. \\ Correspondence to: Yi Yu, MD, PhD; Yigang Li, MD, PhD. Department of Cardiology, Xinhua Hospital affiliated with the School of Medicine, \\ Shanghai Jiao Tong University, 1665 Kongjiang Road, Shanghai 200092, China. Email: yuyi01@xinhuamed.com.cn; liyigang@xinhuamed.com.cn.
}

\begin{abstract}
Background: To assess the diagnostic value of echocardiography in detecting the various types of anomalous origin of the left coronary artery from the pulmonary artery (ALCAPA).

Methods: A total of 30 patients with an established diagnosis of ALCAPA were retrospectively analyzed, and classified into infant- $(n=20)$ and adult-type $(n=10)$ groups according to the age of symptom manifestation and the mode of presentation. All patients underwent echocardiography examination.

Results: Twenty-four out of thirty patients were diagnosed with ALCAPA by echocardiography. The remaining six cases were confirmed by dual-source computed tomography (DSCT) and angiocardiography, respectively. In the infant-type group, there was negligible or no collateral flow between the right coronary artery (RCA) and the left coronary artery (LCA). Eighteen of these patients had enhanced echogenicity of left ventricular (LV) papillary muscles, different degrees of mitral regurgitation (MR) and the RCA to aortic annulus ratio $(\mathrm{RCA} / \mathrm{AO})$ was $>0.12$. In the adult-type group, all ten patients had RCA dilation and significant development of collateralization from the RCA to the dilated LCA. They all had mild MR and RCA/AO was $>0.20$. Preoperatively, left ventricular ejection fraction (LVEF) was significantly lower in infant-type group than in adult-type group $(46.24 \% \pm 5.47 \%$ vs. $61.43 \% \pm 6.38 \%, \mathrm{P}<0.01)$. Cardiac surgery significantly improved post-operative LVEF $(60.12 \% \pm 6.02 \%, \mathrm{P}<0.01 v s$. pre-operation) in infant-type group.

Conclusions: Echocardiography plays a pivotal role in detecting ALCAPA. Imaging and clinical features differ significantly between infant- and adult-type cases.
\end{abstract}

Keywords: Echocardiography; anomalous origin of the left coronary artery from the pulmonary artery (ALCAPA); infant; adult

Submitted Oct 02, 2019. Accepted for publication Dec 13, 2019.

doi: $10.21037 /$ jtd.2020.01.28

View this article at: http://dx.doi.org/10.21037/jtd.2020.01.28

\section{Introduction}

Anomalous origin of the left coronary artery from the pulmonary artery (ALCAPA), also known as BlandWhite-Garland syndrome, is a rare congenital heart defect occurring approximately in 1 out of 300,000 births (1). ALCAPA can be classified into infant and adult types according to the age of symptom onset and the mode of symptom presentation (2). Patients who do not develop significant intracoronary collaterals soon after birth are considered to have infant-type ALCAPA, which is linked with a mortality rate nearing $90 \%$ if untreated (3). The patients affected present with failure to thrive and 
may develop myocardial ischemia, left ventricular (LV) dysfunction, mitral insufficiency and sudden death (3). Adulttype ALCAPA is rare and patients have well-established intracoronary collaterals and patients often do not experience consequence of overt myocardial ischemia (4). Malignant arrhythmias leading to sudden death are common, and $90 \%$ of such patients die suddenly at a mean age of 35 years (5). Early diagnosis and timely surgical intervention aiming to restore a two-coronary-artery circulatory system could bring out excellent outcomes (6).

Echocardiography plays a pivotal role in the detection of ALCAPA (7) by revealing the absence of LCA ostium in the aortic root, the shunting flow from the LCA ostium to the pulmonary artery (2), and the collaterals within the ventricular septum and free ventricular wall (8). Multidetector CT and magnetic resonance imaging are also valuable noninvasive imaging modalities for diagnosing ALCAPA (9-11). The main drawbacks of CT angiography are the relatively high radiation dose required and the inability to assess flow. Meanwhile, magnetic resonance imaging does not clearly display the distal coronary artery, especially in infants, and requires long examination time. Invasive coronary angiography (CAG) remains the gold standard for ALCAPA diagnosis $(12,13)$.

It has been argued that the incidence of ALCAPA is underestimated due to lack of knowledge as well as misdiagnosis. Various imaging modalities have been described, which are helpful on the diagnosis of ALCAPA, mostly in the form of case reports $(6,14)$. Moreover, differences on echocardiographic features between the two ALCAPA types have not been systematically demonstrated.

The aim of the present study was to assess the value of echocardiography in detecting infant- and adult-type ALCAPA, and we summarized echocardiographic features of infant- and adult-type ALCAPA and analyzed the potential causes of misdiagnosis and missed diagnosis.

\section{Methods}

\section{Study population}

Thirty patients with an established diagnosis of ALCAPA in our hospital between August 1997 and August 2018 were retrospectively analyzed. Two patients who died preoperatively and two others lost to follow-up were excluded. The present study was performed under clinical research protocols in accordance with the 1975 Declaration of Helsinki, and approved by the Ethics Committee of
Xinhua Hospital affiliated to Shanghai Jiao Tong University School of Medicine (No. XHEC-D-2018-080).

\section{Preoperative clinical examination}

Patients were evaluated for symptoms of exercise intolerance by the New York Heart Association (NYHA) functional classification based on medical history, stenocardia, history of myocardial infarction, and other diseases. The age of patients at diagnosis and time (in years) from diagnosis to the surgical procedure were analyzed.

\section{Electrocardiography}

A 12-lead electrocardiogram was obtained from each patient. Abnormalities on the electrocardiogram, including abnormal Q-waves, axis deviation, LV hypertrophy, and intraventricular conduction disorders, were analyzed.

\section{Imaging protocol and analysis}

In general, patients were performed echocardiography before and after operation in the period of hospitalization, then followed up in the outpatient. Two-dimensional echocardiography (2DE) was performed on a commercially available system (Philips SONOS iE33, Aliso Viejo, USA; Philips SONOS 5500) equipped with a broadband S5-1 transducer (1-5 MHz) and S8-3 transducer (3-8 MHz). Sedation was required for uncooperative children by oral administration of chloral hydrate (50 to $65 \mathrm{mg} / \mathrm{kg}$ ). Twodimensional echocardiography located access from the parasternal, subcostal, suprasternal, and apical notch windows, with the patient in left lateral decubitus position, was obtained. Left coronary artery (LCA), right coronary artery (RCA) and pulmonary artery imaging served as the focus of echocardiography examination. Echocardiographers in our hospital acquired the data according to a uniform and predetermined protocol, which included display of the coronary artery. Coronary artery dilation was defined as $>2 \mathrm{~mm}$ diameter in infants, $>3 \mathrm{~mm}$ diameter in children at 1-6 years of age, and $>4 \mathrm{~mm}$ in children $>6$ years of age, and $>6 \mathrm{~mm}$ in adults (15).

Meanwhile, development of collateralization, collateral circulation volume, echo enhancement in the LV papillary muscle, LV size, LV ejection fraction (EF), the degree of mitral regurgitation (MR) and the RCA to aortic annulus ratio (RCA/AO) were also assessed (15) for each patient. 
LV asynergies were defined as segmental wall motion abnormalities in the anterior, high-lateral, or apical regions of the left ventricle. The internal dimensions of the coronary artery were measured as well. The internal diameter of the aorta was assessed on the same view in the axial plane.

We performed plain and enhanced DSCT (Somatom Definition, Siemens Medical Solutions, Forchheim, Germany) or CAG (Artis Zee, Siemens, Munich, Germany) (15) to patients in whom echocardiography fails to detect coronary abnormalities. After written informed consent was obtained from each patient, we conducted DSCT scanning or CAG. All images were analyzed for the coronary artery by two experienced cardiologists blinded to all other data. In case of disagreement between the two examiners, a third experienced cardiologist was involved until a consensus was reached.

We divided the patients into two groups based on the imaging characteristics at which they presented with ALCAPA, the group comprising the infant-type who has inadequate collaterals, and the group comprising the adulttype who has adequate collaterals.

\section{Statistical analysis}

Normally distributed continuous variables were presented as mean \pm standard deviation (SD), and those with skewed distribution as median and interquartile range. Categorical variables were presented as frequency and percentage. Differences in continuous and categorical variables were assessed by Student's $t$-test and the Chi-square test, respectively. $\mathrm{P}<0.05$ was considered statistically significant. All statistical analyses were performed with the SPSS 16.0 (v16.0, IBM Corp., Armonk, NY, USA) software.

\section{Results}

\section{Study population and clinical characteristics}

A total of 30 subjects (18 males and 12 females), aged from 1 month to 51 years, were recruited in this study. The ALCAPA patients included 20 infant- and 10 adulttype cases. The general characteristics of participants are summarized in Table 1. Median age was 4 (range, 0.08-9) years in the infant-type ALCAPA group, and 30 (range, 10-51) years in adult-type ALCAPA group.

Among the 30 patients, five patients complicated with atrial septal defect (ASD), three complicated with patent ductus arteriosus (PDA), one complicated with patent foramen ovale (PFO), and the remaining cases showed single ALCAPA.

The preoperative electrocardiogram revealed signs of the myocardial ischemia in twenty infant-type patients $(\mathrm{Q}$ waves in leads I and aVL in nine patients, high lateral leads I and aVL in six patients, ST-T segment changes in the lateral anterior leads V4-V6 in five patients). There were signs of myocardial ischemia in five adult-type patients $(\mathrm{Q}$ waves in leads I and aVL in three patients; ST-T segment changes in the lateral anterior leads V4-V6 and high lateral leads I and aVL in two patients), and electrocardiogram showed LV hypertrophy in three adult-type patients. Two patients had normal electrocardiogram.

\section{Echocardiographic diagnosis}

ALCAPA diagnosis was initially made by echocardiography in 24 out of the 30 patients (Figure 1). The diagnosis was reflected by an antegrade flow in enlarged RCA, the absence of LCA ostium in the aortic root, and the presence of abnormal retrograde flow in the LCA directed into the main pulmonary artery. Color Doppler imaging revealed turbulent color flow regions (intercoronary collaterals) within the ventricular septum and free ventricular wall. Pulsed-wave Doppler imaging showed increased systolic coronary flow of intercoronary collaterals in ALCAPA.

The remaining six patients (four infant- an two adult-type ALCAPA) had complex echocardiographic findings. One patient showed dilatation of left and right coronary arteries, and two others were first diagnosed with left coronary right ventricular fistula. They underwent echocardiography again, which revealed the absence of LCA ostium in the aortic root and an abnormal origin of the LCA from the pulmonary artery in the first patient; the remaining two cases showed an abnormal retrograde flow in the LCA directed into the main pulmonary artery and abundant collateral vessels within the ventricular septum and free ventricular wall. They were finally confirmed as ALCAPA by CAG. Another patient aged 51 years showed dilatation of left and right coronary arteries, but the ostium of the LCA was not detected clearly by echocardiography, and she was advised to undergo DSCT during hospitalization because of pneumonia. Finally, she was confirmed with adult-type ALCAPA by DSCT. Another two children were diagnosed with dilated cardiomyopathy in other hospitals, and finally confirmed with infant-type ALCAPA by echocardiography in our hospital. 
Table 1 Patient characteristics

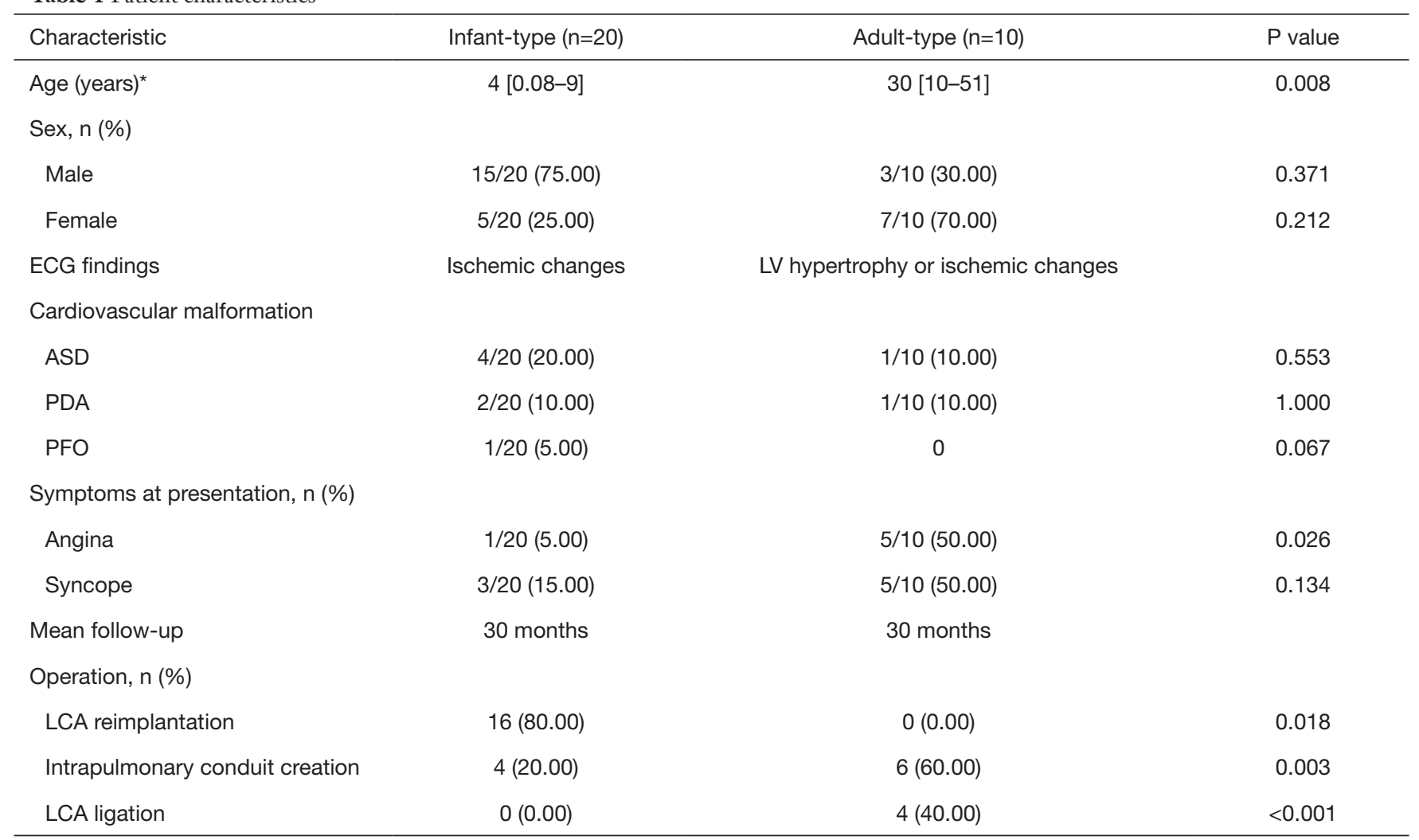

*, data are median [interquartile range]. ECG, electrocardiogram; LV, left ventricular; ASD, atrial septal defect; PDA, patent ductus arteriosus; PFO, patent foramen ovale; LCA, left coronary artery.

\section{Similarities and differences between the infant and adult- type ALCAPA}

The echocardiographic similarities between the two types of ALCAPA were: absence of LCA ostium in the aortic root, anterograde flow in the enlarged RCA, and abnormal retrograde flow in the LCA directed into the main pulmonary artery. Color Doppler imaging revealed turbulent color flow regions (intercoronary collaterals) within the ventricular septum and free ventricular wall. Pulsed-wave Doppler imaging showed increased systolic coronary flow of intercoronary collaterals in ALCAPA.

The echocardiographic differences between the two types of ALCAPA are summarized in Table 2. Compared with adult-type ALCAPA, collateral flow between the RCA and the LCA was not significant or absent in the infanttype group. Eighteen patients had enhanced echogenicity of $\mathrm{LV}$ papillary muscles and twenty showed different degrees of MR. The RCA was mildly dilated. The proximal RCA diameter to the aortic annulus ratio (RCA/AO) exceeded 0.12 in all infant-type patients (Figure 2).
In the adult-type group, ten patients had RCA dilation and significant development of collateralization from the RCA to the LCA; in addition, the RCA was markedly dilated and LCA was also dilated. The patients usually had mild MR and RCA/AO values were $>0.20$, without enhanced echogenicity of LV papillary muscles (Figure 3).

\section{Patient outcomes}

In this patient population, the mean follow-up period was 30 months, excluding four patients who declined surgery. Two patients in the infant-type group were associated with serious heart failure, with one having the largest LV aneurysm and severe MR. Both patients died of heart failure. One patient in the adult-type group denied surgery due to fear of surgery risk. The last patient was asymptomatic, and denied to undergo surgery. The latter two cases were lost to follow-up. Thirty patients underwent cardiac surgery, and were confirmed with ALCAPA perioperatively. In the infant-type group, two children with severe MR and three 

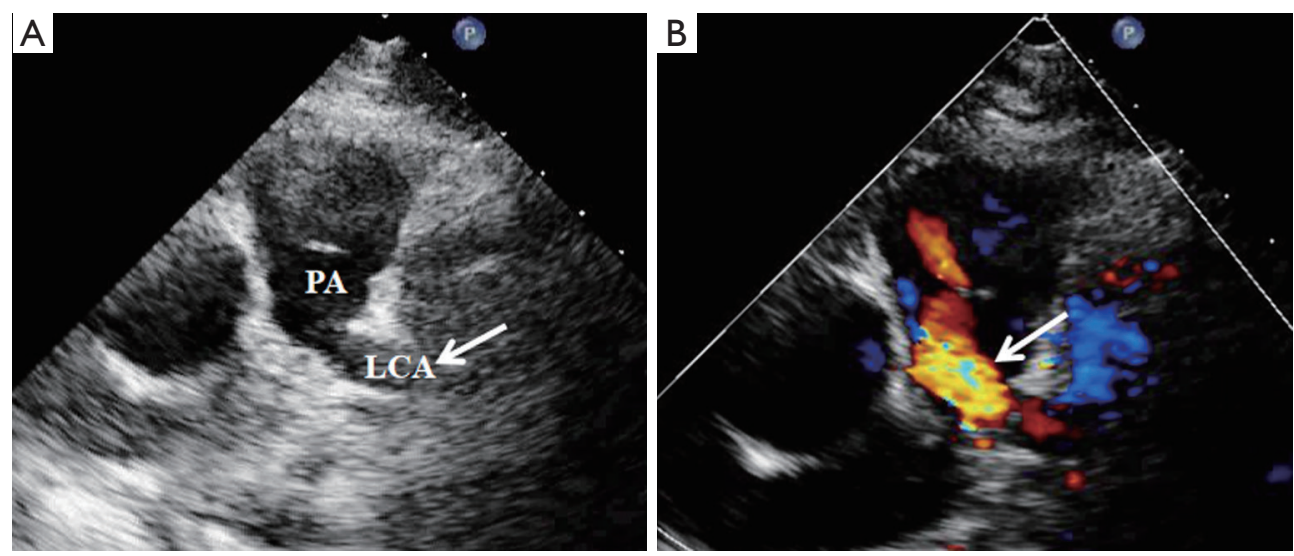

Figure 1 ALCAPA detected by echocardiography. Female, 37 years old, adult-type ALCAPA. (A) Echocardiography showing the left coronary artery originating from the posterior wall of the pulmonary trunk (arrow); (B) shunting flow from the left coronary artery into the pulmonary artery (arrow). ALCAPA, anomalous origin of the left coronary artery from the pulmonary artery; LCA, left coronary artery; PA, pulmonary artery.

Table 2 Differences detected by echocardiography between the infant- and adult-types

\begin{tabular}{|c|c|c|c|}
\hline Parameter & Infant-type $(n=20)$ & Adult-type $(n=10)$ & $P$ value \\
\hline RCA dilated (\%) & $18(90.00)$ & $10(100.00)$ & 0.849 \\
\hline LCA dilated $(\%)$ & $6(30.00)$ & $10(100.00)$ & 0.057 \\
\hline $\mathrm{CA}$ & No advantage & RCA dominance & \\
\hline Collateral circulation volume & Inadequate & Adequate & \\
\hline Enhanced echogenicity of LV papillary muscles (\%) & $18(90.00)$ & 0.00 & 0.018 \\
\hline Wall motion abnormalities & Anterior and lateral wall hypokinesis & None & \\
\hline LV size & Dilated & Normal or mildly dilated & \\
\hline Moderate & $6(30.00)$ & 0.00 & 0.478 \\
\hline Severe & $2(10.00)$ & 0.00 & 0.325 \\
\hline RCA/AO & $>0.12$ & $>0.20$ & \\
\hline
\end{tabular}

ALCAPA, anomalous origin of the left coronary artery from the pulmonary artery; RCA, right coronary artery; LCA, left coronary artery; CA, coronary artery; LV, left ventricular; RCA/AO, right coronary artery to aortic annulus ratio.

children with moderate MR underwent simultaneous mitral valve repair. Mitral valve mobility in three other patients with moderate MR was normal perioperatively, and no treatment for mitral valve was required. The patients with severe MR were improved postoperatively.

Preoperatively, LVEF was significantly lower in infant-type group $(46.24 \% \pm 5.47 \%)$ than in adult-type cases $(61.43 \% \pm 6.38 \%)(\mathrm{P}<0.01)$. LVEF was significantly improved post coronary revascularization $(60.12 \% \pm 6.02 \%)$ $(\mathrm{P}<0.01)$ in infant-type ALCAPA. Meanwhile, postoperative LVEF did not change in adult-type group (Table 3).

Among the 5 patients who underwent mitral valve repair, 

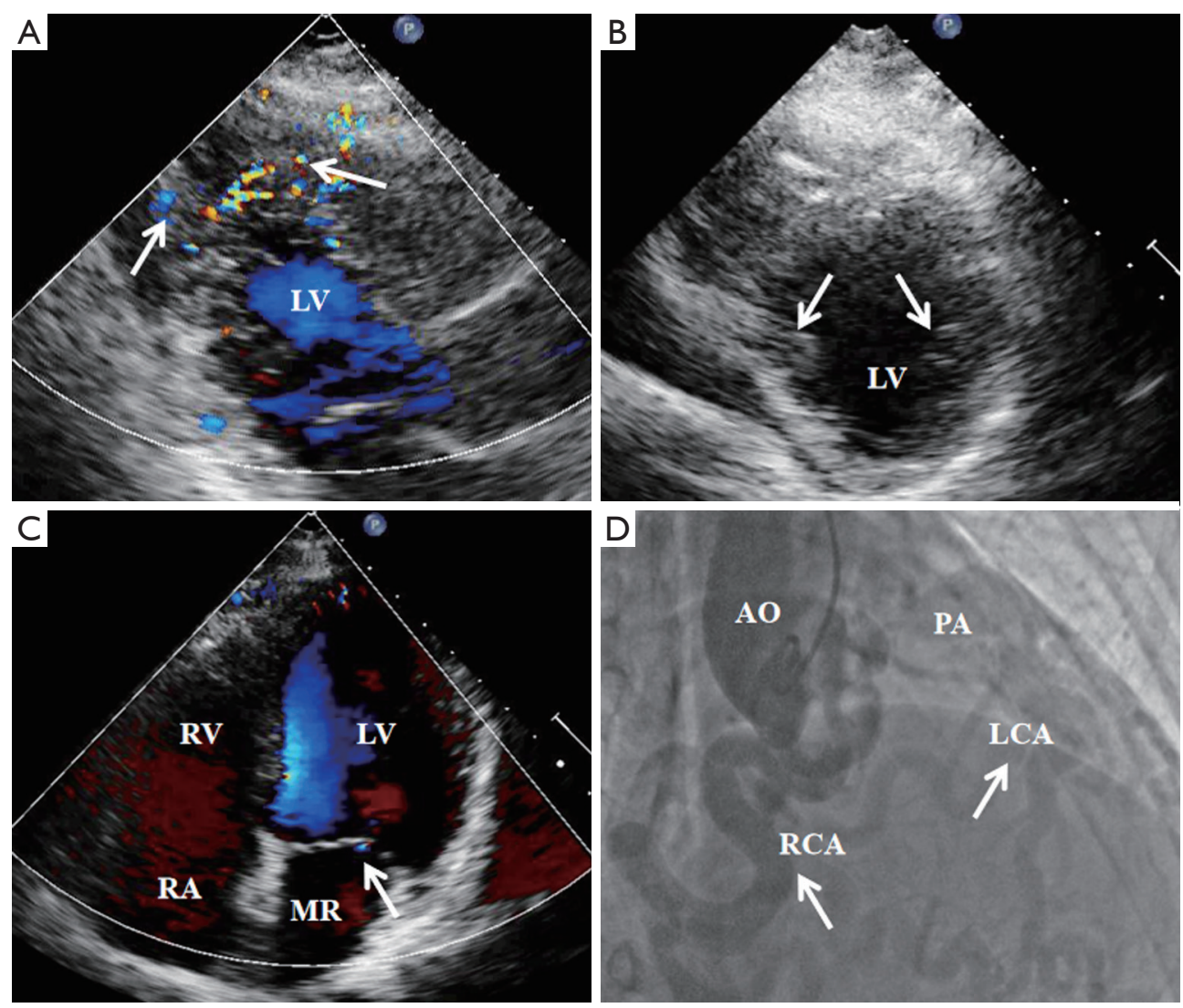

Figure 2 Characteristic findings of infant-type ALCAPA. Male, 1 year old. (A) Less coronary collaterals within the ventricular septum (arrow); (B) echogenic enhancement in LV papillary muscles (arrows), especially in the anterolateral papillary muscle; (C) echocardiography showing left ventricle dilation, and anterior leaflet prolapse of the mitral valve with moderate to severe regurgitation (arrow); (D) coronary angiography showing a slightly dilated right coronary artery (arrow) arising from the aortic sinus, and right coronary supplying collaterals to the LCA (arrow), which drains to the pulmonary artery. ALCAPA, anomalous origin of the left coronary artery from the pulmonary artery; $\mathrm{LV}$, left ventricle; MR, mitral valve regurgitation; RCA, right coronary artery; AO, aorta.

MR became mild.

\section{Discussion}

This study showed that differences between the two types of ALCAPA were presented on intracoronary collaterals, echogenicity of the LV papillary muscle, LV size, LVEF, the degree of MR and RCA/AO. Echocardiography is an excellent method for identifying such differences. Collateral flow between the RCA and the LCA in infanttype ALCAPA was non-significant or absent, leading to myocardial ischemia, myocardial infarction, enhanced echogenicity of $L V$ papillary muscles, $L V$ enlargement and dysfunction, more pronounced MR and mildly increased RCA to AO ratio. In the adult-type ALCAPA, the coronary steal phenomenon was found, with significant development of intracoronary collateralization. Significant RCA and LCA dilatation causes preferential blood flow into the lowpressure pulmonary system rather than the high-resistance myocardium, which could lead to exertional dyspnea, angina and cardiac arrhythmias. Other features included normal LV size and function, mild MR and obviously increased RCA/AO ratio. Preoperatively, the infant-type group showed significantly reduced LVEF compared with adult-type cases. MR in the infant-type group was more serious than in adult-type cases. Postoperatively, LVEF and MR were significantly improved in infant-type patients, and remained unchanged in the adult-type group.

Symptomatic infants usually have significant apical LV dilation and hypokinesis or akinesis, even with ventricular 

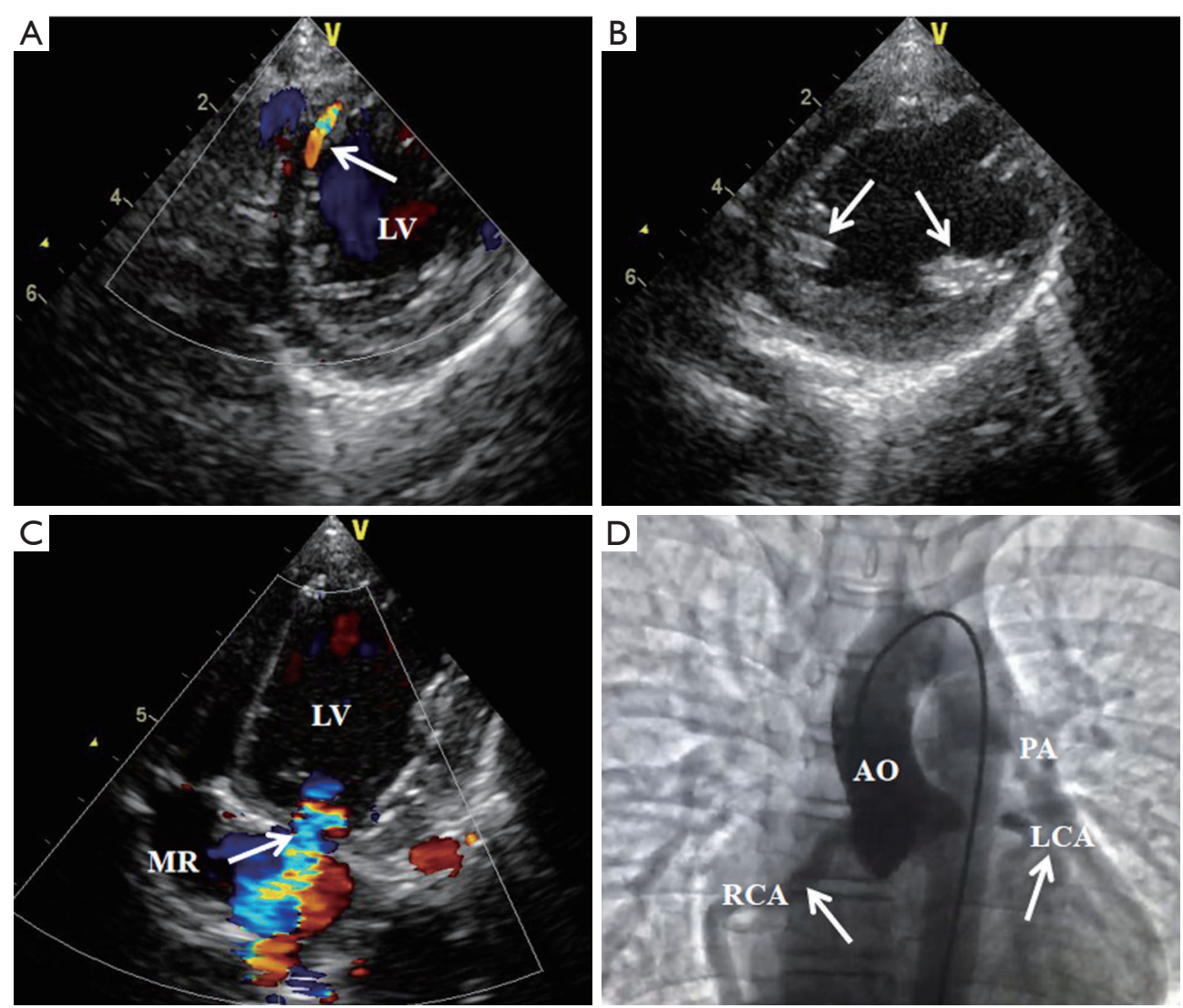

Figure 3 Characteristic findings of adult-type ALCAPA. Female, 37 years old. (A) Parasternal long-axis view, color Doppler imaging revealing multiple coronary collaterals within the ventricular septum and free ventricular wall (arrows); (B) echocardiography at parasternal short axis showing papillary muscles without echogenic enhancement (arrows); (C) echocardiography at the apical 4-chamber view showing the mitral valve with slight regurgitation (arrow); (D) coronary angiography showing a markedly dilated right coronary artery (arrow) arising from the aortic sinus, and the left coronary artery (arrow) being filled from collaterals and shunting into the pulmonary artery. ALCAPA, anomalous origin of the left coronary artery from the pulmonary artery; RV, right ventricle; RA, right atrium.

Table 3 Changes of left ventricular ejection fraction (EF) between the infant- and adult-types before and after operation

\begin{tabular}{lccc}
\hline Ejection fraction $(\%)$ & Infant-type $(n=20)$ & Adult-type $(n=10)$ & $P$ value \\
\hline Before-operation & $46.24 \pm 5.47$ & $61.43 \pm 6.38^{\mathbf{\Delta}}$ & 0.006 \\
Post-operation & $60.12 \pm 6.02^{\mathbf{\Delta}}$ & $63.30 \pm 6.78^{\S}$ & 0.241 \\
\hline
\end{tabular}

Before-operation vs. post-operation: ${ }^{\mathbf{\Lambda}}, \mathrm{P}<0.01$. Infant-type vs. adult-type: ${ }^{\mathbf{\Lambda}}, \mathrm{P}<0.01 ;{ }^{\S}, \mathrm{P}>0.05$.

aneurysm. Gribaa et al. (16) reported a 7-month-old child with ALCAPA who died due to anterior myocardial infarction. This report also presented a 22-month-old child with ALCAPA who had significant LV apical dilation and functional impairment. Thus, symptomatic infants often have a severe clinical course and warrant prompt surgery once diagnosis is established. LV dilation and anterior leaflet prolapse of the mitral valve are the most readily discernable signs of echocardiography; meanwhile, the clinical sign is apical systolic murmur, but could lead to a false diagnosis of dilated cardiomyopathy (16) or congenital mitral valve prolapse. In this situation, dilated RCA and increased RCA/ AO ratio were the helpful signs in distinguishing ALCAPA from dilated cardiomyopathy; echogenic chordae tendineae or the papillary muscle of the mitral valve also indicates an ischemic origin and provides a valuable clue for ALCAPA 
detection (17). In some cases $(18,19)$, the RCA was not dilated, and retrograde flow into the RCA could not be seen. The anomalous LCA was extremely close to the aorta, sometimes could mislead the echocardiographer to judge the LCA as arising normally from the aorta. Moreover, severe pulmonary hypertension (20) and the absence of significant intercoronary collateral arteries added to this challenge. The echocardiographer should thus be aware of two helpful identifiable markers: hyperechogenicity of papillary muscles and abnormal segment activity of $\mathrm{LV}$ wall (LV hypokinesis or akinesis) (20). Previous studies $(11,21,22)$ reported misdiagnosed cases by transthoracic echocardiography in adult patients with ALCAPA. Frigault et al. (23) reported a 45-year-old patient with ALCAPA was masqueraded as peripartum cardiomyopathy for 8 years. LV dilatation, severe MR, pulmonary hypertension, LVEF of $35 \%$ with moderate global hypokinesia served as reasons for the misdiagnosis. In this study, one patient aged 51 years was initially suspected with pneumonia, but echocardiography showed well-developed collaterals within the ventricular septum and the absence of LCA ostium in the aortic root, which prompted us to consider a diagnosis of ALCAPA.

In addition, adult-type ALCAPA with late onset of signs or symptoms is less prone to myocardial ischemia and remains often asymptomatic, associated with a relatively benign prognosis. No segmental ventricular wall motion abnormality was observed in the LCA perfusion territory (anterior septal, anterior and lateral wall) of adult-type ALCAPA cases in the present study, indicating adequate collateral circulation and myocardial perfusion at rest. Those patients can be asymptomatic at the beginning, and can be induced in some specific conditions, such as pregnancy, pneumonia, or even occasionally found during physical examination. The onset of adult-type is late, and exists at all ages. Tian et al. (24) reported ALCAPA in an adult patient who presented with a continuous murmur along the left sternal border, accompanied by multiple collateral vessels between the anterior descending artery and the RCA, with no record of myocardial ischemia within 3 years of follow-up. In some cases, abundant collaterals are often misdiagnosed as coronary artery right ventricular fistula or coronary artery pulmonary artery fistula. Kawasaki disease is a major cause of giant coronary artery, and is easily confused with ALCAPA (25). Our team (26) compared echocardiography and DSCT in the diagnosis of coronary artery aneurysm due to Kawasaki disease: dilated coronary artery and coronary artery aneurysm could be seen in patients with Kawasaki disease, with LCA ostium in the normal location. This was an obvious difference between ALCAPA and Kawasaki disease.

In general, our findings are in line with the reports by Kurup et al. and Belli et al. (20,27), indicating that improvements in cardiac function are more obvious in infant-type cases even in those with severely depressed LV function, because reversibility of the ischemic myocardium after cardiac surgery is higher than that in adult-type patients. Poor postoperative coronary flow involving aneurysms, occlusions and stenoses, or the development of a collateral circulation before surgical repair, may influence LV function recovery in both ALCAPA types.

Echocardiographic imaging of coronary arteries in adult patients can be challenging. Specifically, the origin of LCA ostium is always difficult to detect by two-dimensional echocardiography because of low spatial resolution and poor penetration through the chest wall, creating an artifact mimicking the location of the aortic root. Moreover, careful inspection of coronary arteries is rarely performed in adults. Even when combining two-dimensional echocardiography with Doppler color flow mapping, diagnosis might still be missed in adult patients. Therefore, easily identifiable echocardiographic markers of ALCAPA might be helpful for diagnosis (28). In adult-type ALCAPA, enlarged RCA, collaterals, and abnormal blood flow from the high-pressure RCA artery filling the LCA via extensive collaterals and exiting the LCA into the pulmonary artery could be readily identified by $2 \mathrm{DE}$ and color flow Doppler imaging. Intercoronary collateral vessels are well developed within the ventricular septum, particularly in adult patients. These striking findings strongly indicate an anomalous coronary artery. Additionally, combining echocardiography with multidetector CT angiography (11), especially for adult-type cases, could improve diagnosis. As the gold standard diagnostic modality, CAG is not always necessary in the current era, but is usually reserved for questionable cases before cardiac surgery.

\section{Limitations}

Firstly, this was not a randomized study, and indications for the procedures were dependent both on patient characteristics and the physician's preferences. Secondly, sample size was relatively small, and there might also be time bias because the follow-up period was shorter for the ALCAPA group. It could not divide patients by age group (newborns, 1-5 years, 6-12 years, 13-18 years and adults) according to the echocardiographic characteristics. Thirdly, 
young pediatric patients could not tell clinical symptoms in infant-type ALCAPA, which led to partial deletion of clinical data. However, these limitations may not affect echocardiographic findings. A multicenter study should be performed in the future to address these issues.

\section{Conclusions}

Scrupulous echocardiographic examinations with color Doppler and Doppler flow imaging are essential for accurate diagnosis of lately discovered ALCAPA. Meanwhile, collateral circulation, echogenicity of the LV papillary muscle, LV size, LVEF, the degree of MR and RCA/AO ratio differ between infant- and adult-type ALCAPA.

\section{Acknowledgments}

We acknowledge the work of clinical doctors involved in the discovery of the case.

Funding: This work was supported by Shanghai Science and Technology Committee Clinical Field Project (grant: 17411954800). Shanghai Health and Family Planning Commission Foundation (grant: 201740136).

\section{Footnote}

Conflicts of Interest: The authors have no conflicts of interest to declare.

Ethical Statement: The authors are accountable for all aspects of the work in ensuring that questions related to the accuracy or integrity of any part of the work are appropriately investigated and resolved. The present study was performed under clinical research protocols in accordance with the 1975 Declaration of Helsinki, and approved by the Ethics Committee of Xinhua Hospital affiliated to Shanghai Jiao Tong University School of Medicine (No. XHEC-D-2018-080).

Open Access Statement: This is an Open Access article distributed in accordance with the Creative Commons Attribution-NonCommercial-NoDerivs 4.0 International License (CC BY-NC-ND 4.0), which permits the noncommercial replication and distribution of the article with the strict proviso that no changes or edits are made and the original work is properly cited (including links to both the formal publication through the relevant DOI and the license). See: https://creativecommons.org/licenses/by-nc-nd/4.0/.

\section{References}

1. Erdinc M, Hosgor K, Karahan O. Repair of anomalous origin of the left coronary artery arising from right pulmonary artery with rolled-conduit-extended reimplantation in an adult. J Card Surg 2011;26:604-7.

2. Kanoh M, Inai K, Shinohara T, et al. Outcomes from anomalous origin of the left coronary artery from the pulmonary artery repair: long-term complications in relation to residual myocardial abnormalities. J Cardiol 2017;70:498-503.

3. Wesselhoeft H, Fawcett JS, Johnson AL. Anomalous origin of the left coronary artery from the pulmonary trunk. Its clinical spectrum, pathology, and pathophysiology, based on a review of 140 cases with seven further cases. Circulation 1968;38:403-25.

4. Yau JM, Singh R, Halpern EJ, et al. Anomalous origin of the left coronary artery from the pulmonary artery in adults: a comprehensive review of 151 adult cases and a new diagnosis in a 53-year-old woman. Clin Cardiol 2011;34:204-10.

5. Takemoto K, Hirata K, Tanimoto T, et al. Combined noninvasive doppler echocardiography and coronary computed tomography lead to diagnosis of anomalous left coronary artery from the pulmonary artery(ALCAPA) syndrome. Circ J 2015;79:1136-8.

6. Mavroudis C. Anomalous origin of the left coronary artery from the pulmonary artery presenting in adulthood: a French nationwide retrospective study an editorial commentary. Semin Thorac Cardiovasc Surg 2017; 29:491-3.

7. Jiang GP, Wang HF, Gong FQ, et al. Diagnostic value of parasternal pulmonary artery short-axis view for the anomalous origin of the left coronary artery from the pulmonary artery. J Cardiol 2014;63:444-8.

8. Blanco VM, Blalock SE, Ramaciotti C, et al. Oxygen supplementation is helpful for the echocardiographic detection of anomalous left coronary artery from the pulmonary artery. J Am Soc Echocardiogr 2010;23:1099-102 .

9. Chow BJ, Wells GA, Chen L, et al. Prognostic value of 64-slice cardiac computed tomography severity of coronary artery disease, coronary atherosclerosis, and left ventricular ejection fraction. J Am Coll Cardiol 2010;55:1017-28.

10. Komócsi A, Simor T, Tóth L, et al. Magnetic resonance studies in management of adults cases with Bland-WhiteGarland syndrome. Int J Cardiol 2007;123:e8-11.

11. Zhu J, Liu Y, Zhu SB, et al. Anomalous Origin of the left coronary artery from the pulmonary artery confirmed by 320-Slice Computed Tomography. Pediatr Cardiol 
2013;34:1283-4.

12. Díaz-Zamudio M, Bacilio-Perez U, Herrera-Zarza MC, et al. Coronary artery aneurysms and ectasia, role of coronary CT angiography. Radiographics 2009;29:1939-54.

13. Sun Z, Lin C, Davidson R, et al. Diagnostic value of 64-slice CT angiography in coronary artery disease: a systematic review. Eur J Radiol 2008;67:78-84.

14. Drinkovic N, Margetic E, Smalcelj A, et al. Echocardiographic diagnosis of anomalous origin of the left coronary artery from the pulmonary artery. Eur J Echocardiogr 2008;9:309-10.

15. Patel SG, Frommelt MA, Frommelt PC, et al. Echocardiographic diagnosis, surgical treatment, and outcomes of anomalous left coronary artery from the pulmonary artery. J Am Soc Echocardiogr 2017;30:896-903.

16. Gribaa R, Slim M, Ben Salem H, et al. Anomalous origin of the left coronary artery from the pulmonary artery presenting as dilated cardiomyopathy: a case report. J Med Case Rep 2014;8:170.

17. Tutar E, Uysalel A, Nacar N, et al. Anomalous origin of the left coronary artery from the main pulmonary artery detected on echocardiographic screening study of newborns. Int J Cardiol 2004;97:561-2.

18. Malakan Rad E. Anomalous origin of left coronary artery from pulmonary artery associated with pulmonary hypertension. Indian Heart J 2014;66:700-3.

19. Varghese M, Kothari S. The caveats in the diagnosis of anomalous origin of left coronary artery from pulmonary artery (ALCAPA). Images Paediatr Cardiol 2010;12:3-8.

20. Kurup RP, Daniel R, Kumar RK. Anomalous origin of the left coronary artery from the pulmonary artery in infancy with preserved left ventricular function: Potential pitfalls and clues to diagnosis. Ann Pediatr Cardiol

Cite this article as: Yu Y, Wang QS, Wang XF, Sun J, Yu LW, Ding M, Li YG. Diagnostic value of echocardiography on detecting the various types of anomalous origin of the left coronary artery from the pulmonary artery. J Thorac Dis 2020;12(3):319-328. doi: 10.21037/jtd.2020.01.28
2008;1:65-7.

21. Fuchs MM, Le RJ, Cullen MW, et al. Multimodal imaging of anomalous left coronary artery from the pulmonary artery in a 75-year-old woman. Tex Heart Inst J 2017;44:395-8.

22. Esmaeilzadeh M, Hadizadeh N, Noohi F. Anomalous origin of the left coronary artery from the pulmonary artery (ALCAPA) in an old adult. J Tehran Heart Cent 2011;6:148-51.

23. Frigault J, Lafrenière-Bessi V, Perron J, et al. Anomalous left coronary artery from the pulmonary artery: masquerading as peripartum cardiomyopathy. Ann Thorac Surg 2018;106:e33-5.

24. Tian Z, Fang LG, Liu YT, et al. Anomalous origin of the left coronary artery from the pulmonary artery detected by echocardiography in an asymptomatic adult. Intern Med 2013;52:233-6.

25. Yamamura K, Sakamoto I, Yamasaki Y, et al. Silent progression of coronary artery thrombosis in a pregnant woman with anomalous origin of left coronary artery from the pulmonary artery. Circ J 2017;81:1236-7.

26. Yu Y, Sun K, Wang RF, et al. Comparison study of echocardiography and dual-source CT in diagnosis of coronary artery aneurysm due to Kawasaki disease. Echocardiography 2011;28:1025-34.

27. Belli E, Roussin R, Ly M, et al. Anomalous origin of the left coronary artery from the pulmonary artery associated with severe left ventricular dysfunction: results in normothermia. Ann Thorac Surg 2010;90:856-60.

28. Selmi K, Bergaoui H, Boujnah MR. Echocardiographic marker for bland-white-garland syndrome in adult. J Am Soc Echocardiogr 2011;24:1056.e1-4. 